\title{
Internato de enfermagem na atenção básica: desafios da sua efetividade
}

\author{
Basic care nursing internship: challenges for it to be effective \\ Internado de enfermería en la atención básica: desafíos de su eficacia
}

\author{
Heluana Cavalcante Rodrigues'; Maria Socorro de Araújo Dias"; Antônia Eliana Araújo Aragão"li; \\ Maria Adelane Monteiro da Silvalv; Diógenes Farias Gomesv"; Maria da Conceição Coelho Britovı
}

\begin{abstract}
RESUMO
Objetivo: analisar os desafios para a efetividade da formação do internato em enfermagem na Atenção Básica, a partir dos discursos coletivos dos atores envolvidos. Método: estudo exploratório-descritivo, que ocorreu entre março de 2013 e janeiro de 2015 . Teve sua investigação no Internato de Enfermagem na Atenção Básica de duas Universidades do Nordeste brasileiro. Participaram sete discentes, três docentes e seis preceptores, selecionados a partir de critérios pré-estabelecidos. Utilizou o Discurso do Sujeito Coletivo, com o apoio do Quantiqualisoft (C). Resultados: foram identificados, como principais desafios para a efetividade do internato, a interligação entre teoria e prática e a integração ensino-serviço, as quais foram entendidas como desafios do seio educacional e estrutural, o que denotou a necessária participação do sistema de saúde pública. Conclusão: evidenciou-se a necessidade de reestruturação dos internatos, destacando a construção de uma agenda de compromisso e deveres entre universidade e gestão municipal.
\end{abstract}

Descritores: Internato de enfermagem; Atenção primária à saúde; Educação em enfermagem; Ensino superior.

\section{ABSTRACT}

Objective: to examine the challenges for nursing education in Basic Care to be effective, based on the collective discourses of the actors involved. Method: this exploratory, descriptive study of Basic Care nursing internships took place between March, 2013, and January, 2015, at two universities in northeast Brazil. Participants were seven students, three teachers and six preceptors, selected on pre-established criteria. Collective Subject Discourse was used with the support of Quantiqualisoft $\subset$ software. Results: the main challenges for an internship to be effective were identified as being the interconnection between theory and practice, and integration between education and work. These were understood as challenges of an education and structural order, denoting necessary participation by the public health system. Conclusion: there was shown to be a need to restructure internships, particularly to construct an agenda of commitment and duties between university and municipal administration.

Descriptors: Nursing internship; Primary health care; Education, nursing; Education, higher.

\section{RESUMEN}

Objetivo: analizar los desafíos para la efectividad de la formación en enfermería en la Atención Básica, a partir de los discursos colectivos de los atores involucrados. Método: estudio exploratório y descriptivo, que ocurrió entre marzo de 2013 y enero de 2015. Su investigación tuvo lugar en el Internado de Enfermería en la Atención Básica de dos Universidades del Nordeste brasileiro. Participaram siete alumnos, tres profesores y seis preceptores, seleccionados por criterios pre-establecidos. Se utilizó el Discurso del Sujeto Coletivo con el apoyo de QuantiqualisoftC. Resultados: se identificaron como principales desafíos para una efectividad del internado, la interconexión entre la teoría y la práctica, y la integración del enseñanza-servicio, que fueron entendidas como desafíos del seno educativo y estructural, lo que mostró necesaria participación del sistema de salud pública. Conclusión: se mostró la necesidad de reestructuración de los internados, destacando una construcción de una agenda de compromiso y deberes entre la universidad y la gestión municipal.

Descriptores: Prácticas de enfermeira; Atención primaria de salud; Educación en enfermería; Educación superior.

\section{INTRODUÇÃO}

A Constituição Federal (CF) de 1988, em seu artigo 200, esclarece a necessidade da formação de recursos humanos para o Sistema Único de Saúde (SUS). Dessa forma, os princípios doutrinários instituídos neste sistema (equidade, universalidade e integralidade) influen- ciaram a constituição de novos arranjos educacionais, a fim de desenvolver profissionais aptos para um novo modelo de assistência à Saúde ${ }^{1,2}$.

Mediante a proposta, somou-se o desafio de (re)construir o ensino na Saúde, buscando refutar o

Enfermeira. Mestre em Saúde da Família pela Universidade Federal do Ceará (UFC). Tutora do Sistema Saúde Escola de Sobral. Escola de Formação em Saúde da Família Visconde de Sabóia. Ceará, Brasil. E-mail: heluanacavalcante@yahoo.com.br

"Enfermeira. Pós-doutoramento em Cuidados Clínicos pela Universidade Estadual do Ceará (UECE). Docente do Curso de Enfermagem. Universidade Estadual Vale do Acaraú (UVA).Ceará, Brasil. E-mail: socorroad@gmail.com

"'Enfermeira. Doutora em Enfermagem pela UFC. Coordenadora do Curso de Enfermagem. Instituto Superior de Teologia Aplicada. Ceará, Brasil. E-mail: antoniaelianearaujo@gmail.com

IVEnfemeira. Doutora em Enfermagem pela UFC. Coordenadora do Curso de Enfermagem. Universidade Estadual Vale do Acaraú. Ceará, Brasil. E-mail: adelanemonteiro@hotmail.com

vEnfermeiro. Mestrando em Saúde da Família pela UFC. Bolsista demanda social CAPES. Universidade Federal do Ceará. Ceará, Brasil. Ceará, Brasil. E-mail: diogenesfgo@gmail.com V'Enfermeira. Mestre em Saúde da Família pela UFC. Assistente de Pesquisa do Núcleo de Ensino e Pesquisa. Escola de Formação em Saúde da Família Visconde de Sabóia. Ceará, Brasil. E-mail: marycey@hotmail.com 
modelo de ensino biomédico, intensificar a formação significativa e o desenvolvimento de competências ${ }^{2}$. Com esta ideia, iniciaram-se os movimentos de reforma do ensino na Saúde, promovendo a articulação entre o SUS e instituições de ensino.

Na década de 1990, elementos normativos intensificaram o ensino na Saúde. Destaca-se a Lei das Diretrizes e Bases da Educação Nacional (LDB), que estabelece uma reorientação do conceito de educação afirmando que: "abrange os processos formativos que se desenvolvem na vida familiar, na convivência humana, no trabalho, nas instituições de ensino e pesquisa, movimentos sociais e organizações da sociedade civil e manifestações culturais" ${ }^{3}$. Tais aspectos têm sido influenciados por medidas provisórias, a exemplo da $n$ ㅇ 746, de 22 de setembro de 2016, que altera o conteúdo da LDB com ênfase ao ensino técnico e exclusão de disciplinas como Filosofia e Sociologia ${ }^{3}$. Nessa perspectiva, a educação intensifica a adoção de novos modelos curriculares, o que desencadeia a formação de diretrizes para cada curso, permitindo interpretar as concepções de campo e núcleo ${ }^{4}$.

Nessa trajetória, consolidam-se, ainda na década de 1990, as Diretrizes Curriculares Nacionais (DCN) dos cursos da Saúde, as quais emergem na tentativa de incorporar a atuação humanística, os princípios do SUS e a sensibilidade aos problemas advindos do contexto social. Destaca-se, para este estudo, que as DCN do Curso de Enfermagem almejam a formação de um profissional generalista, orientado para a atenção à saúde da população, fortalecimento das relações profissionais e da educação permanente ${ }^{5}$.

Os objetivos da formação na Enfermagem instituem também um aspecto reflexivo sobre o fazer profissional para o SUS. Nessa ótica, o internato em Enfermagem configura-se como uma etapa fundamental para o desenvolvimento de habilidades e atitudes necessárias para o exercício da profissão. As vivências proporcionadas nesta etapa da graduação possibilitam o contato com a realidade do sistema de saúde, gerando significado, a partir da prática assistida por um preceptor ${ }^{6,7}$.

Torna-se, então, importante uma investigação que compreenda o internato em Enfermagem enquanto etapa da formação do enfermeiro para atuação no SUS, tencionando reflexões que permeiam os desafios imbuídos para a efetividade dessa estratégia.

Do exposto, delineia-se, nesse estudo, o objetivo de analisar os desafios para a efetividade da formação do internato em Enfermagem na Atenção Básica, a partir dos discursos coletivos dos atores envolvidos.

\section{Metodologia}

Estudo de epistem qualitativa, exploratório-descritivo, realizado de março de 2013 a janeiro de 2015, em duas Instituições de Ensino Superior (IES) - uma pública e uma privada - do município de Sobral, Ceará, no Nordeste brasileiro. Essas instituições foram selecionadas a partir dos seguintes critérios: atender a região norte do estado, ofertar o curso bacharelado em enfermagem, e estar inserida no campo de práticas da atenção básica do município.

Participaram profissionais e discentes envolvidos no internato em Enfermagem do segundo semestre letivo de 2014, sendo sete discentes, três docentes e seis preceptores. A delimitação do quantitativo de participantes respeitou a técnica de saturação de informações ${ }^{8}$. Esses participantes estiveram distribuídos nos territórios da Estratégia Saúde da Família do Alto do Cristo, Coelce, Padre Palhano e Sumaré.

Os critérios de elegibilidade dos participantes foram: discente, ter cursado no mínimo $75 \%$ da carga horária total do internato; docente, estar colaborando com o Internato na Atenção Básica; preceptor, possuir experiência anterior nesta função, ser um profissional do serviço de atenção básica e estar acompanhando um dos discentes das IES matriculados no internato.

As informações foram coletadas mediante entrevista semiestruturada, com questionamentos sobre o internato em Enfermagem na Atenção Básica, a articulação teoria e prática, integração ensino-serviço e as competências apreendidas pelos discentes como reflexo de um currículo instituído para a formação por competências no SUS.

O conteúdo das entrevistas foi organizado no Quantiqualisoft (C) e analisado com a técnica da análise do Discurso do Sujeito Coletivo (DSC). Estes aspectos metodológicos foram adequados para este estudo na medida em que se possibilitou um discurso-síntese dos participantes, representando uma escolha interessante para estudos qualitativos ${ }^{9}$.

Estudo aprovado por um Comitê de Ética em Pesquisa, sob Parecer no 842.449/2014. A identificação dos discursos está assim representada: DSCdoc, para docentes; DSCprec, para preceptores; e DSCdisc, para discentes.

\section{RESUltAdos E Discussão}

Os resultados do estudo sinalizam dois desafios para a efetividade da formação no internato na $A B$ : a relação teoria e prática e a integração ensino-serviço. $O$ primeiro toma uma conotação educacional (relação com o ensino-aprendizagem) e o segundo, como estrutural, ao envolver aspectos que transcendem a formação em Enfermagem e englobam universidade, serviços de saúde e gestão na Saúde.

A relação de proximidade de discentes, docentes e preceptores com o fenômeno investigado permitiu uma interpretação homogênea. Com isso, os discursos são apresentados nas categorias: "Teoria e prática no internato de Enfermagem da Atenção Básica: base para discussão dos desafios"; e "A integração ensino-serviço: intercâmbio para efetividade do internato de Enfermagem". 
Teoria e prática no internato de Enfermagem da Atenção Básica: base para discussão dos desafios

Ao considerar toda a trajetória permeada por avanços, potencialidades e fragilidades no ensino e na Saúde brasileira, pretendeu-se, com esta análise, compreender o quão próximo ou distante a formação dos enfermeiros, para a realidade do SUS, se apresenta nestas duas IES, sendo prospectivo a realidade de outras instituições brasileiras.

o currículo foi delineado para que o aluno tenha o tempo todo teoria e prática de forma articulada. Eles levam para o campo o que eles adquiriram na teoria para o desenvolvimento da prática, contribuindo para o fortalecimento das equipes que eles estão inseridos. Eles estão indo muito embasado teoricamente. (DSCdoc)

O DSCdoc permite a reflexão dos itinerários formativos adotados nos cursos de Enfermagem das IES. Os cursos analisados depreendem uma construção histórica regional atrelada às necessidades sociais e de saúde vigentes, contribuindo para uma trajetória curricular dinâmica. Esta característica conota uma relação sócio-histórica da construção desses cursos com as necessidades de saúde regionais.

A Enfermagem, historicamente, apresenta uma trajetória vasta que revela a adoção de estratégias formativas essencialmente técnicas por um longo tempo, orientada pelas necessidades do sistema de saúde público vigente ${ }^{10}$. Para este estudo, verificou-se que o modelo curricular utilizado é destacado como uma ferramenta atualizada e importante para o alinhamento entre teoria e prática, fundamental para o processo de ensino-aprendizagem do discente de Enfermagem. Dessa forma, a necessária relação entre teoria e prática apresentou-se como um aspecto essencial para concatenar a formação do enfermeiro às necessidades da Atenção Básica.

O currículo é, se não, um anseio pela efetiva relação entre teoria e prática. Em um dos seus conceitos, possibilita o conhecimento dos extremos da teoria e prática, o que destaca um continuum que merece ser desbravado nos contextos educacionais ${ }^{11}$.

Consonante, é importante que a relação teoriaprática seja um elemento apresentado na proposta curricular dos Cursos como aspecto essencial para a efetividade da formação. Assim, o internato em Enfermagem enseja uma aprendizagem significativa, sustentada na vivência dos discentes em dispositivos e serviços da Atenção Básica. Dessa maneira, o mesmo pôde ser visualizado como uma estratégia de enfrentamento para o constante dilema inserido na relação teoria e prática.

Para a concretização dessa relação, vem sendo deflagradas iniciativas com vistas a estreitar o vínculo das universidades com os serviços de saúde e até mesmo entre outros cursos, como segue a proposta em um estudo anterior, ao suscitar uma formação inovadora em
Terapia Ocupacional, a partir de um currículo integrado e interdisciplinar ${ }^{12}$.

O modelo curricular adotado nos cursos tem possibilitado a qualidade no ensino dos discentes, tomando, como indicador, o conhecimento teórico e seus reflexos na prática. Para tanto, é referido que esse processo de aprendizagem é iniciado nos semestres anteriores, sendo amadurecido durante o internato:

O aluno desde o primeiro semestre vivencia simultaneamente a teoria e prática (DSCdoc).

O DSCdoc apresenta que a práxis da teoria e prática do ensino se manifesta desde os períodos iniciais do curso. No entanto, ressalta-se que, nos momentos iniciais da graduação em Enfermagem, as disciplinas/módulos são essencialmente teóricas e estruturantes para os momentos posteriores do curso. Contudo, foram identificadas duas perspectivas pedagógicas para os cursos, bancária na IES privada e humanista na pública, o que podem ser atribuídas as diferenciações também adotadas por outras instituições situadas no território nacional.

As DCN da Graduação em Enfermagem são claras ao suscitar a formação de um profissional generalista, humanista, crítico e reflexivo, para tanto, que necessita dotar-se de conhecimento a respeito da atenção à saúde, tomada de decisões, comunicação, liderança, administração e gerenciamento e educação permanente, o que permite inferir a necessidade de conhecimentos teóricos básicos que fundamentam uma prática segura ${ }^{13}$.

Sustentado nas discussões de Paulo Freire, destaca-se ainda que ainda existirá uma técnica efetiva que possibilite a relação entre teoria e prática totalmente ${ }^{14}$. Isso revela os constantes desafios que se encontram nessa relação e, nessa hora, também justifica as fragilidades encontradas nos internatos analisados, como vestígios do modelo dicotômico entre teoria e prática.

Apoiando-se no DSCdoc, salienta-se que os processos de apreensão do conhecimento, a partir das vivências na graduação, são etapas necessárias para a compreensão da prática profissional do enfermeiro. Isto se revela como um movimento necessário para o amadurecimento do discente enquanto futuro profissional.

Ademais, é possível identificar que o internato de Enfermagem revela, aos discentes, situações para as quais não foram preparados na graduação. Esta vivência faz-se necessária para desencadear uma aprendizagem significativa, catalisando o desejo pela investigação de novas situações a serem vivenciadas no dia a dia do profissional da Atenção Básica.

O exposto corrobora-se com o DSCdisc, o qual apresenta que estas situações despertam habilidades que serão necessárias para sua atuação no sistema de saúde orientado pelo SUS:

Na prática você acaba aprendendo muito mais que na teoria. Tem algumas coisas que são novas. A saúde é 
muito dinâmica. A gente aprende uma coisa e quando você vai para a realidade é outra coisa completamente diferente (DSCdisc).

O DSCdisc apresenta um descompasso na relação teoria e prática. 0 mesmo salienta, além da dificuldade com algumas situações do cotidiano, fatos desconexos com a realidade da prática aprendida na universidade. Há, no entanto, duas hipóteses interpretativas para esta relação de desafio: a primeira é a falta de insumos necessários para a realização dos procedimentos nos serviços de saúde que corroborem com uma atenção com qualidade e efetiva; e a segunda se assenta na desproporcionalidade entre o ensino da graduação com a realidade do profissional no serviço.

O exposto se sustenta na realidade de que os serviços de Atenção Básica têm enfrentado desafios estruturais para uma atenção resolutiva, e apontam divergências nas formações em enfermagem em instituições pública e privada. Este desafio poderia ser superado, caso a Atenção Básica ocupasse um espaço na agenda prioritária dos gestores e que fosse encarada como uma estratégia que ordena o cuidado no SUS. Para além disso, a construção dos itinerários formativos apresenta certa dissonância em relação aos serviços dispostos no SUS, aspecto que contrapõe o artigo 200 da CF de $1988^{15}$.

As hipóteses apresentadas revelam que os aspectos organizativos do Sistema de Saúde e de Ensino são pontos que merecem ser discutidos como prioritários para uma formação qualificada, como demonstrado a seguir:

Muitas coisas nós vimos pela primeira vez no internato, como preencher aquela ficha nos casos de tuberculose, existem coisas muito diferentes (DSCdisc).

Assim, o discente percebe que o ambiente de prática consolida o saber teórico e aproxima a discussão de casos, a partir da realidade dos serviços de saúde. No entanto, resgatam a difícil interconexão entre teoria e prática.

Os DSC's possibilitaram refletir o internato como um espaço que possibilita o desenvolvimento de competências para o cuidado ampliado na Atenção Básica, fomentando uma formação orientada pela ética, postura profissional e corresponsabilização.

\section{A integração ensino-serviço: intercâmbio para efetivi- dade do internato de Enfermagem}

Para que a formação atenda às necessidades de saúde da população, se faz necessária uma maior aproximação entre estes dois contextos historicamente desconectados, para que os atores envolvidos possam compreender os aspectos relacionados à vida real e aos espaços de prestação de cuidado de saúde. O discurso, a seguir, mostra pontos de fragilidades nesta articulação.

Normalmente no internato não sou comunicada, pelo menos a mim enquanto enfermeira eu não fico sabendo, só quando eles chegam ou então só liga, eu estou mandando os internos tal dia, muitas vezes os alunos vêm é só, o preceptor, o coordenador do estágio não vem aqui passar para gente, explicar as atividades que eles vão ter que desenvolver, o período do internato. O que nos repassam é assim: até tal dia tem que entregar o portfólio, tal dia tem que apresentar o estudo de caso.(DSCprec)

O DSCprec sinaliza aspectos que tornam a integração ensino-serviço um desafio de gestão de processo. Este binômio curto, porém, complexo, impulsiona reflexões que envolvem a gestão do ensino na Saúde e o papel docente. Outrora, permite dimensionar a afetação de profissionaispreceptores diante desta dificuldade e as estratégias de enfrentamento que têm sido desenvolvidas.

Além da necessidade de alinhamento entre teoria e prática, o DSC dos participantes revelou o desafio da integração ensino-serviço, apontando incidentemente a pouca comunicação entre os profissionais das IES e os profissionais-preceptores dos serviços.

Explica-se que, neste estudo, adotou-se o termo "profissional-preceptor", alinhado à política do município que defende o Sistema de Saúde como um sistema saúde-escola. Neste, os profissionais colaboram na formação profissional da Saúde ${ }^{16}$.

O DSC dos preceptores reforça a dificuldade indicada ao salientar que há necessidade de intensificar a presença de docentes supervisores que facilitem determinados momentos de aprendizagem diante dos quais têm dificuldades de eleger a melhor abordagem metodológica e teórica que possa suprir a necessidade de formação dos discentes na Atenção Básica.

Essa comunicação normalmente não é regular entre o serviço e a IES, entre os preceptores da atenção básica e a IES, se dando mais no início e no final do internato. [...] as vezes vinha um profissional pegar as fichas para gente fazer a avaliação, não perguntava como foi o andamento do processo, o que precisava ser melhorado. Esporadicamente, temos contato com o professor orientador, ou seja, tem turmas que a gente tem contato, outras não.(DSCprec).

O discurso aponta para um diálogo que necessita ser repensado no intuito de estreitar os vínculos entre o ensino e serviço. Nessa premissa, o diálogo e reflexão tornam-se oportunos para efetivo desenvolvimento dos discentes, o que aponta para as ponderações sobre complementariedade que deve permear todo o processo de ensino-aprendizagem, minimizando a formação de lacunas de ensino ${ }^{17}$.

Essa realidade é amenizada quando se observa, no DSCprec, a maneira como a relação se estabelece quando apresenta uma representação da IES nos serviços de saúde.

No caso da outra IES, o professor orientador [refere-se ao preceptor da IES] vem até a unidade e tem um contato regular com o preceptor enfermeiro da unidade de saúde. Nós não temos no internato corpo docente suficiente. Não temos nos articulado de modo que os professores possam acompanhar esses alunos como a gente gostaria, dentro do município. (DSCprec) 
O representante, apontado no discurso como "professor orientador", assume o papel de preceptor do curso, que atua no acompanhamento dos discentes nos serviços, em parceria com profissionais-preceptores que atuam na $A B$. Tal característica, disponível apenas em uma das IES, foi apontada como potencialidade para a relação entre ensino e serviço, a qual oportuniza o melhor acompanhamento do discente.

No mesmo discurso, o profissional sinaliza que a dificuldade que baliza esse processo é a deficiência de docentes que oportunizem a efetividade do internato. Nessa medida, verifica-se que a relação ensino-serviço envolve, também, a concretização de um corpo docente que garanta a distribuição equânime para o monitoramento do processo ensino-aprendizagem realizado em campo.

A integração ensino-serviço foi sempre citada nos discursos dos preceptores e encarada na perspectiva desafiadora de conectar efetivamente as instâncias do ensino superior com os serviços e profissionais do sistema público de Saúde. Vislumbra-se, portanto, pela consolidação de canais comunicativos, que aparentam relativa facilidade para serem formulados e instituídos, ao se considerar os avanços concretizados no Sistema Saúde Escola de Sobral.

O evidente anseio pela melhoria da integração entre o ensino e serviço são perspectivas também peculiares de estudos disponíveis na literatura científica que, semelhantes a este, tratam o internato de Enfermagem como foco da discussão. Dessa maneira, salienta-se que esta integração deve influenciar na organização de espaços que possibilitem a qualificação para o atendimento, promovendo um intercâmbio de saberes entre discentes, docentes e profissionais do serviço na construção de um perfil profissional comprometido com a qualidade na Saúde ${ }^{18}$.

Conforme indicado no discurso dos profissionaispreceptores, os docentes de uma das IES indicam, também, uma dificuldade no monitoramento e avaliação das atividades do internato na Atenção Básica, quando evidenciam que a avaliação conta com a participação (efetiva) de docentes, na condução de portfólios e estudos de caso, e dos profissionais-preceptores, na realização da avaliação final do discente, porém, de maneira separada. Esta dificuldade é tida como um desafio operacional, aparentemente superado pela outra IES, na medida em que dispõe de profissionais preceptores contratados e disponíveis para o acompanhamento dos discentes em campo.

Um dispositivo que tem sido utilizado para facilitar e qualificar a integração ensino-serviço, observado neste estudo, foi a presença de uma escola para a formação de trabalhadores da área da Saúde vinculada ao munícipio que vem possibilitando a organização e alocação dos discentes no sistema local de Saúde e possibilitando um canal de comunicação entre a universidade e os serviços de Saúde. Esta característica faz parte do processo de planejamento do internato. O DSC dos docentes afirma esta iniciativa:
Sabendo quais são os alunos que vão para atenção básica, a gente entra em contato com a Escola de Saúde, faz o pedido formal, solicitando os campos de prática. A gente chama os alunos, faz uma reunião e aí a gente vai junto com eles planejar, a partir do dimensionamento que a gente tem, realizando um sorteio para a divisão deles no campo. Depois a gente explica para os alunos os objetivos do internato na atenção básica, as competências que eles têm que ter, as responsabilidades deles, apresenta o instrumento, discute como vão ser avaliados e caso não julguem justo, a gente muda. (DSCdoc)

Ainda, o mesmo discurso evidencia que as competências são formuladas a partir de iniciativas das universidades, configurando como uma prática pertinente aos docentes, sem suscitar a participação dos profissionais-preceptores. Vale destacar a abertura para alterações dos processos avaliativos, o que sugere uma perspectiva democrática da participação do discente.

Em alguns momentos do discurso dos docentes, é possível identificar que a causa para a não inclusão dos profissionais-preceptores nos processos organizativos do internato se dá pela pouca disponibilidade destes em relação a um cronograma de reuniões com os docentes, conforme se evidencia:

A gente tentou marcar momentos com os preceptores do serviço e nós enquanto supervisores para poder ajustar o instrumento de avaliação, acabou que a gente não fez. Tentamos várias vezes, mas não tinha agenda, não tinha pauta, foi difícil. E dizer assim, a culpa foi do Sistema, não! A culpa foi muito mais nossa de não ter insistido mais. (DSCdoc)

Os discursos das duas instituições possibilitam uma reflexão acerca do sistema saúde-escola, qual seja: a indisponibilidade de agenda (tempo) e envolvimento dos profissionais-preceptores com o processo de aprendizagem dos internos nos serviços de saúde, quando os momentos de planejamento e avaliação ocorrem extra-horário e espaço de trabalho, devendo suscitar novas estratégias.

Ainda se salienta a necessidade de estabelecer um responsável para a ocorrência deste desencontro em momentos que envolvam discentes, profissionais-preceptores e docentes. Para tanto, devem estar dispostos recursos humanos e estruturais que permitam afirmar sua potencialidade para a efetividade do internato. A relação integral do Sistema de Saúde engloba, além dos docentes, discentes e preceptores, gestores do ensino e Sistema de Saúde ${ }^{19}$. Para tanto, torna-se essencial o envolvimento destes numa relação ímpar, a fim de propiciar um ensino qualificado para profissionais da Saúde.

Nessa perspectiva, a integração ensino-serviço configurou-se como um dos desafios para a efetivação do internato de Enfermagem na Atenção Básica como estratégia de formação para o SUS que, unido aos desafios da relação teoria-prática, evidencia a necessária formação de iniciativas/estratégias de enfrentamento para estes problemas. 


\section{CONCLUSÃO}

Este estudo, ao centrar-se em uma etapa da formação do curso de graduação em Enfermagem, possibilita reflexões mediante o modelo de ensino aplicado e os desafios estruturais nos itinerários formativos.

Ao adscrever a realidade de um município do Nordeste brasileiro, torna-se uma relevante evidência para a comunidade científica, na possiblidade de fomentar uma possível análise comparativa entre os desafios encontrados com a realidade de outros cursos.

Os resultados identificados a partir da utilização do DSC são claros ao apontar que a opinião entre os sujeitos é homogênea. Para tanto, compreende-se que os desafios apontados tornam-se singulares mediante o papel ocupado pelos discentes, docentes e preceptores, sendo afetados a partir das suas singularidades de inserção no ensino e aprendizagem.

É evidente a necessidade de reestruturações no internato analisado. Quanto à teoria e prática, é importante a compreensão e aproximação do ensino da universidade com os desafios dos serviços de Saúde, com o cuidado de não intensificar uma prática dissociada da ciência. Na integração ensino-serviço, são necessários recursos profissionais que possibilitem a melhoria na relação entre universidade e serviço, mesmo que estratégias já tenham sido implantadas. Desse modo, é necessária a construção de uma agenda que componha compromissos e deveres entre Universidades e sistema de Saúde.

Recomenda-se a realização de estudos que fundamentem a formação do enfermeiro na Atenção Básica considerando todo o processo formativo da Graduação contando, como problematização, a integração ensinoserviço e a relação teoria-prática.

\section{REFERÊNCIAS}

1. Presidência da República (Br), Casa Civil, Subchefia para Assuntos Jurídicos. Constituição da República Federativa do Brasil de 1988[Internet]. Brasília (DF): Presidência da República; 1988 [cited 2015 May 11]. Available from: https://www.planalto.gov. br/ccivil_03/constituicao/constituicao.htm

2. Seriano KN, Muniz VRC, Carvalho MEIM. Percepção de estudantes do curso de Fisioterapia sobre sua formação profissional para atuação na atenção básica no Sistema Único de Saúde. Rev Fisioter pesqui [Internet]. 2013 July/Sept [cited 2015 May 11];20(3):250-5. Available from: http://www.scielo.br/pdf/fp/ v20n3/09.pdf

3. Presidência da República (Br), Casa Civil, Subchefia para Assuntos Jurídicos. Lei no 9.334, de 20 de dezembro de 1996. Estabelece as diretrizes e bases da educação nacional. [Internet]. Brasília (DF): Presidência da República; 1996 [cited 2015 May 11]. Available from: https://www.planalto.gov.br/ccivil_03/Leis/L9394.htm

4. Campos GWS. Saúde pública e saúde coletiva: campo e núcleo de saberes e práticas. Ciênc saúde coletiva [Internet]. 2000 [cited 2015 May 11];5(2):219-30. Available from: http://www.scielo.br/ $\mathrm{pdf} / \mathrm{csc} / \mathrm{v} 5 \mathrm{n} 2 / 7093 . \mathrm{pdf}$

5. Ministério da Educação (Br), Conselho Nacional de Educação, Câmara de Educação Superior. Resolução CNE/CES no 3, de 7 de novembro de 2001. Institui Diretrizes Curriculares Nacionais do Curso de Graduação em Enfermagem [Internet]. Brasília (DF):
Ministério; 2001 [cited 2014 Feb 12]. Available from: http://portal. mec.gov.br/cne/arquivos/pdf/CESO3.pdf

6. Lima TC, Paixão RC, Cândido EC, Campos CJG, Ceolim MF. Estágio curricular supervisionado: análise da experiência discente. Rev. bras. enferm. [Internet]. 2014 Jan/Feb [cited 2015 May 11];6(7):133-40. Available from: http://www.scielo.br/pdf/reben/ v67n1/0034-7167-reben-67-01-0133.pdf

7. Acioli S, Kebian VA, Faria MGA, Ferraccioli P, Correa VAF. Prática de cuidado: papel do enfermeiro na atenção básica. Rev. enferm. UERJ [Internet]. 2014 Sept/Oct [cited 2015 May 11];22(5):62742. Available from: http://www.epublicacoes.uerj.br/index.php/ enfermagemuerj/article/view/12338/12288

8. Fontanella BJB, Ricas J, Turato ER. Amostragem por saturação em pesquisas qualitativas em saúde: contribuições teóricas. Cad. saúde pública [Internet]. 2008 Jan [cited 2015 May 11];24(1):1727. Available from: http://www.scielo.br/pdf/csp/v24n1/02.pdf 9. Figueiredo MZA, Chiari BM, Goulart BNG. Discurso do sujeito coletivo: uma breve introdução à ferramenta de pesquisa qualitativa. Distúrb comun [Internet]. 2013 Apr [cited 2015 May 11]; 25(1):129-36. Available from: http://revistas.pucsp.br/index.php/ dic/article/view/14931/11139

10. Lazzari DD, Pedro ENR, Sanches MO, Jung W. Estratégias de ensino do cuidado em enfermagem: um olhar sobre as tendências pedagógicas. Rev. gaúch. enferm[Internet]. 2011 Dec [cited 2015 May 11];32(4):688-94. Available from: http://www.scielo.br/pdf/ rgenf/v32n4/v32n4a08.pdf

11. Young M. Teoria do currículo: o que é por que é importante. Cad pesqui [Internet]. 2014 [cited 2015 May 11];44(151):190-202. Available from: http://publicacoes.fcc.org.br/ojs/index.php/cp/ article/view/2707/2659

12. Barba PCSD, Silva RF, Joaquim RHVT, Brito CMD. Formação inovadora em terapia ocupacional. Interface comum saúde educ [Internet]. 2012 July/Sept [cited 2015 May 11];16(42):82942. Available from: http://www.scielo.br/pdf/icse/v16n42/ v16n42a19.pdf

13. Fortuna V. A relação teoria e prática na educação em Freire. REBES [Internet]. 2015 Oct/Dec [cited 2015 May 11];1(2):64-72. Available from: https://seer.imed.edu.br/index.php/REBES/article/download/1056/746

14. Cavalcanti PCS, Oliveira Neto AV, Sousa MF. Quais os desafios para qualificação da Atenção Básica na visão dos gestores municipais? Saúde Debate [Internet]. 2015 Apr/June [cited 2015 May 11]:39(105):323-36. Available from: http://www.scielo.br/pdf/ sdeb/v39n105/0103-1104-sdeb-39-105-00323.pdf

15. Martins Junior, Parente JRF, Sousa FL, Sousa MST. A residência multiprofissional em as de da família de Sobral-Ceará. Sanare (Sobral, Online) [Internet]. 2008 July/Dec [cited 2015 May 11];7(2):23-30. Available from: https://sanare.emnuvens.com. $\mathrm{br} /$ sanare/article/viewFile/28/23

16. Rodrigues AGF, Carvalho Neto ERG. Eu aprendo, tu aprendes, ele aprende: em busca de uma prática docente mais democrática na EJA. Rev FAEEBA [Internet]. 2012 Jan/June [cited 2015 May 11]:21(37):127-36. Available from: http://www.revistas.uneb.br/ index.php/faeeba/article/viewFile/469/404

17. Baldoino AS, Veras RM. Análise das atividades de integração ensino-serviço desenvolvidas nos cursos de saúde da universidade federal da Bahia. Rev. Esc. Enferm. USP. [Internet]. 2016 [cited 2015 May 11];50(esp):17-24. Available from: http://www.scielo. br/pdf/reeusp/v50nspe/pt_0080-6234-reeusp-50-esp-0017.pdf 18. Gonçalves CNS, Corrêa AB, Simon G, Prado ML, Rodrigues J, Reibnitz KS. Integração ensino-serviço da voz de profissionais de saúde. Rev. enferm. UFPE on line 2014 [cited 2016 Jan 14];8(6):1678-86. Available from: http://www.revista.ufpe.br/ revistaenfermagem/index.php/revista/article/view/5969

19. Autonomo FROM, Hortale VA, Santos GB, Both SHO. A preceptoria na formação médica e multiprofissional com ênfase na atenção primária - análise das publicações brasileiras. Rev bras educ méd. 2015; 39(2):316-27; 2015. 